\title{
DISEÑO DE UN SISTEMA DE GESTIÓN DE INVENTARIOS PARA EL ALMACÉN TÉCNITALLER S.A.S DE LA CUIDAD NEIVA-HUILA, COLOMBIA
}

\section{DESIGN OF AN INVENTORY MANAGEMENT SYSTEM FOR TÉCNITALLER S.A.S. WAREHOUSE IN NEIVA-HUILA, COLOMBIA}

\author{
Héctor Andres Hernández ${ }^{1} *$ (D); Yeimi Lorena Cruz-Gil ${ }^{2}$ (D); María Daniela Puentes-Saavedra ${ }^{3}$ (D); \\ Diego Ernesto Mendoza-Patiño ${ }^{4}$ (D). \\ 1. Universidad Nacional Abierta y a Distancia, Colombia. andreshernandez19@gmail.com \\ 2. Universidad Antonio Nariño, Colombia. ycruz352@uan.edu.co \\ 3. Universidad Antonio Nariño, Colombia.mpuentes77@uan.edu.co \\ 4. Universidad Antonio Nariño, Colombia.diego.mendoza@uan.edu.co
}

*Autor de correspondencia: Héctor Andres Hernández, email: andreshernandez19@gmail.com

\section{RESUMEN}

La siguiente investigación tuvo como fin diseñar un sistema de gestión de inventarios para el almacén Técnitaller S.A.S de la ciudad de Neiva; esto debido a la ineficiente administración de sus inventarios los cuales han generado reprocesos, faltantes, obsolescencia, bajo de nivel de servicio, daño en mercancías entre otros; la metodología empleada corresponde a un estudio no experimental- transversal y descriptivo que inicia con el diagnóstico del área, la elaboración de la propuesta de gestión de inventarios y el análisis económico de la misma; como resultado del análisis se determinó que el modelo a implementar en la empresa integra un sistema de información que comprende 4 módulos de gestión (Compras, almacén, inventarios y despacho), cuyo valor de implementación es de \$21.595.150 el cual será realizado por Word Office. La implementación de este sistema permitirá a la empresa optimizar la gestión de inventarios la cual se verá reflejada en unos menores costos de mantener el inventario, de pedir, de almacenar entre otras variables.

Palabras clave: Inventario; Existencia; Clasificación ABC; Rotación; Gestión de Inventarios.

Cómo citar:

Hernández, Héctor Andres; Cruz-Gil, Yeimi Lorena; Puentes-Saavedra, María Daniela; Mendoza-Patiño, Diego Ernesto. (2021). Diseño de un sistema de gestión de inventarios para el almacén técnitaller S.A.S de la cuidad Neiva-Huila, Colombia. Revista de Investigaciones Universidad del Quindio, 33(2), 143-152. https://doi. org/10.33975/riuq.vol33n2.562 


\section{ABSTRACT}

The following research had the purpose of designing an inventory management system for the warehouse Técnitaller S.A. S of the city of Neiva; this due to the inefficient administration of its inventories which have generated reprocesses, shortages, obsolescence, low level of service, damage in merchandise among others; the methodology used corresponds to a non-experimental, transversal and descriptive study that begins with the diagnosis of the area, the elaboration of the proposal of inventory management and the economic analysis of the same one; As a result of the analysis it was determined that the model to be implemented in the company integrates an information system comprising 4 management modules (Purchasing, warehouse, inventories and dispatch), whose implementation value is $\$ 21.595 .150$, which will be implemented by Word Office. The implementation of this system will allow the company to optimize inventory management which will be reflected in lower costs of maintaining inventory, ordering, storing and other variables.

Keywords: Inventory; Stock; ABC Classification; Rotation; Inventory Management.

\section{INTRODUCCIÓN}

El correcto orden y administración de inventarios es uno de los principales factores que contribuyen a la gestión operativa y misional de una organización, como a la gestión de servicio al cliente; esto permite obtener un mejor desempeño a nivel de servicio y en su dinámica financiera para el área responsable de garantizar el flujo de productos. De acuerdo con lo anterior es fundamental que las organizaciones cuenten con las herramientas necesarias para lograr mantener un óptimo sistema de inventarios.

Según (Bravo, 1981) el seguimiento a los inventarios es una variable de incidencia directa en la disminución de costes en las empresas, por ende, debe considerarse, a tal punto que una óptima intervención asegure un mejor comportamiento financiero para la organización. Para la empresa, para llevar a cabo este proyecto se implementará fundamentos de ingeniería industrial que permitirá identificar, analizar los procesos que faciliten el diseño de estrategias orientadas a fomentar y solucionar situaciones no deseadas que se presenten.

Para mitigar y mejorar las condiciones que generan esa problemática, se estableció como objetivo el diseño de un Sistema de Gestión de Inventarios a partir de la implementación de un software integrado por 4 módulos que van desde la compra, el almacenamiento, el inventario y la distribución (Gutiérrez \& Vidal, 2007, pág. 138).

Técnitaller S.A.S, es una empresa dedicada a la prestación de servicios de reparación, mantenimiento y venta de repuestos para vehículos en la ciudad de Neiva; el almacén, en los últimos años ha tenido un ineficiente manejo del inventario de repuestos, con lo cual a través de la matriz DOFA Y VESTER aplicadas se identificaron las principales problemáticas que llevaban a la empresa a tener bajos niveles de servicio de atención al cliente, problemas de faltantes, obsolescencia entre otros.

De acuerdo con lo anterior, se plantea como propósito diseñar un sistema para la gestión de inventarios que permita optimizar el movimiento y manejo de los productos en el almacén Técnitaller S.A.S ubicado en la ciudad de NeivaHuila, lo cual permita establecer las necesidades de abastecimiento, así como la de mantener un stock de seguridad, en función de la demanda.

El presente artículo se divide en 3 secciones. En la primera se describe la metodología empleada para 
la identificación de los problemas de Técnitaller S.A:s en el manejo de los inventarios- La segunda los resultados obtenidos del diagnóstico situacional de la empresa objeto de estudio. En la tercera se muestran las conclusiones del análisis de la información.

\section{METODOLOGIA}

\section{Tipo y enfoque de la investigación}

La presente investigación a través del tipo de diseño no experimental- transversal y se aplicó un tipo de estudio descriptivo, lo cual permitió analizar las variables de estudio mediante observación con el fin de identificar la forma como se desarrollan las diferentes actividades que implican procesos de inventario como despachos, alistamientos, pedidos, almacenamiento, atención al cliente, tiempos de espera entre otros; las cuales permitieron realizar propuestas de mejoramiento de las entradas, salidas y existencias de la mercancía, así como también su organización y almacenamiento, a fin de aplicarse un modelo de control de inventario en la empresa Técnitaller S.A.S; para esto se empleó la técnica de observación directa y entrevista con el gerente y los trabadores de la empresa que intervienen en las diferentes actividades.

\section{Recolección y análisis de datos}

Para el proceso de recolección de datos se planteó una matriz de recolección de datos de cada uno de los objetivos propuestos en el proyecto como se indica a continuación en la tabla 1 .

Tabla 1. Método de recolección de datos

\begin{tabular}{lllc}
\hline \multicolumn{1}{c}{ Objetivo } & Que se va a hacer & Como se va a hacer & $\begin{array}{c}\text { Con que } \\
\text { herramienta }\end{array}$ \\
\hline $\begin{array}{l}\text { 1- Elaborar un diagnóstico de los } \\
\text { métodos e instrumentos utilizados } \\
\text { para llevar el inventario en el almacén } \\
\begin{array}{l}\text { Técnitaller S.A.S. } \\
\text { Realizar un diagnostico }\end{array}\end{array}$ & $\begin{array}{l}\text { Realizando una descripción de la } \\
\text { situación actual como se manejan } \\
\text { los inventarios en el almacén }\end{array}$ & $\begin{array}{c}\text { MTESTER, lista de } \\
\text { chequeo }\end{array}$ \\
\hline
\end{tabular}
2- Elaborar la propuesta del sistema Proponer un modelo A partir de la combinación de de gestión inventarios para el de Gestión (Rincón M, almacén Técnitaller S.A.S 2016). punto de reorden, $\mathrm{ABC}$, lote económico, lead time entre otros
Hoja de simulación en Excel

Hoja de simulación financiera para
3- Realizar el estudio económico de Realizar un análisis la implementación de la propuesta de viabilidad de la del sistema de gestión de inventarios inversión de mejora para el almacén Técnitaller S.A.S

(Orosco Fabián, 2009).
Establecer las tasas de retorno de la inversión, así como la VAN y TIR del proyecto proyectos de inversión

Elaboración propia

\section{RESULTADOS}

\section{Diagnóstico del proceso}

\section{Lista de chequeo por proceso}

Para realizar el diagnóstico sobre manejo de inventarios en la empresa Técnitaller S.A.S se diseñó una lista de chequeo la cual arrojó los siguientes datos como se muestra en la tabla 2. 
Tabla 2. Lista de chequeo

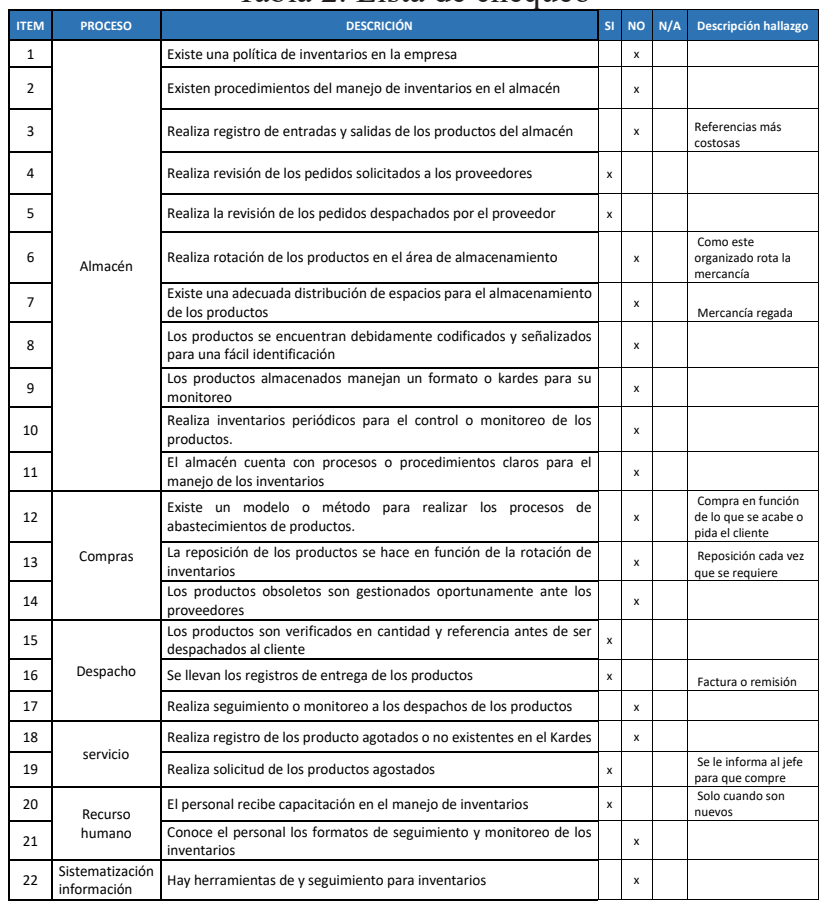

Elaboración propia

De acuerdo con los datos obtenidos de la tabla 2 se pudo determinar que:

- Proceso de almacenamiento: No hay una política de inventarios, por ende, no se tienen procedimientos de gestión de almacén, ni se evidencia registros para el control y monitoreo de entradas y salidas.

- Diagnostico proceso compras: No se evidencia modelo para abastecimiento de productos, por ende, la reposición de productos sigue un patrón en función de los agotados.

- Diagnostico proceso de despacho: No hay un control en el flujo de referencias para reparaciones.

- Diagnostico proceso de servicio: No se realiza un registro de productos agotados.

- Diagnóstico del proceso de recurso Humano: Aunque la rotación de personal no es alta, no existe una política de entrenamiento y capacitación, para personal nuevo.

- Diagnóstico del proceso de sistematización de la información: No se evidencio un sistema de información ni hojas de Excel para el registro y seguimiento de los inventarios.

En conclusión, no se evidenciaron procesos de trazabilidad, ni controles en puntos críticos del proceso de gestión de inventarios.

\section{Diagnostico DOFA}

Con relación al análisis interno y externo que se realizó a la empresa aplicando la herramienta de análisis DOFA (Debilidades, Oportunidades, Fortalezas y Amenazas) se obtuvieron los siguientes resultados como se muestra en la tabla 3.

Tabla 3. Análisis DOFA

\section{DEBILIDADES OPORTUNIDADES}

1.No existe política de inventarios

2.No hay control y monitoreo en rotación de inventarios

3. No se registran entradas y salidas de mercancía

\section{Agotados}

5.Almacenamiento descentralizado

6.No hay patrón de marcación de mercancía

7.No hay un método para cálculo de reposición de mercancía

8.No hay

Procedimientos sobre control de inventarios

9. Mercancía obsoleta
1.Establecer un modelo para la administración de inventarios

2.Reorganizar el sistema de gestión de almacenes

3.implementacion de un sistema que le permita controlar y monitorear a nivel de inventarios sus operaciones 4.Protocolizar procesos y procedimientos

5.Negociar con proveedores la mercancía obsoleta

6. Actualización de listas de referencias

7. Diseño de un plan de entrenamiento y formación 
10. Proceso

de formación,

entrenamiento de

tipo informal sin

registro

FORTALEZAS AMENAZAS

1. La empresa

cuenta con más

de 30 años de

experiencia en el

mercado.

2. Actualmente

posee clientes

fidelizados.

3.Servicios de

reparación y

mantenimiento

de vehículos con

calidad

4.Cuenta equipos

e infraestructura

suficiente para

garantizar la calidad

y eficiencia de sus

operaciones

5.Cuenta con un

capital humano

integral, competitivo

y comprometido

para administración

de sus operaciones.

6.Excelente vida

financiera y

crediticia

7.Ubicación

estratégica

5. PQR del proceso.

2. La pandemia que incide en la demanda de servicios.

\section{Clientes cambien el taller por otro}

4. Estrategias de la competencia en cuanto a servicio y disponibilidad de repuestos

\section{Daño mercancía expuesta a la intemperie}

$$
\text { servicios. }
$$

proceso, abastecimiento agotado, entre otros; con lo cual se procede a diseñar las estrategias, como se describen en la tabla 4 análisis FODA.

Tabla 4. Análisis FODA

\section{ESTRATEGIAS(FO) ESTRATEGIAS(DO)}

1-5-Negociar
con proveedores
mercancía obsoleta

4-1. Rediseño de áreas

de almacenamiento

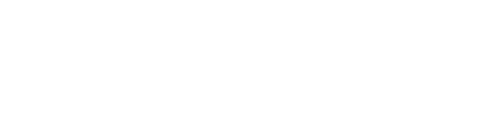

1-3. Cubrir las

expectativas mediante una óptima gestión de disponibilidad o consecución de repuestos para clientes
1,2,3-1.3,6-Diseñar o elaborar un modelo de inventarios que establezca una política clara, eficiente y adecuada

4,7-3,4. Implementar un método de reposición de inventarios

5,6-2,3,4,6-Reingenieria de gestión física de almacenes y mercancía

8,10-7. Plan de formación continua 9-5. Gestión proveedores

4-3. Agotados reducen la oportunidad de servicio por ende la expectativa del cliente

\begin{tabular}{ll} 
& $\begin{array}{l}\text { 8,10-7. Plan de } \\
\text { formación continua } \\
\text { 9-5. Gestión } \\
\text { proveedores }\end{array}$ \\
\hline ESTRATEGIAS(FA) & ESTRATEGIAS(DA) \\
\hline $\begin{array}{l}\text { 1-1 Modelo de } \\
\text { planeación y gestión } \\
\text { de servicio }\end{array}$ & $\begin{array}{l}\text { 4-3. Agotados reducen } \\
\text { la oportunidad de } \\
\text { servicio por ende la } \\
\text { 2-3 Optimización }\end{array}$ \\
$\begin{array}{l}\text { de servicios para } \\
\text { generar Benchmarking del cliente } \\
\text { competitivo }\end{array}$ & \\
$\begin{array}{l}\text { 3-3 Renegociar } \\
\text { con otro proveedor } \\
\text { los precios de los } \\
\text { productos. }\end{array}$ & \\
3-4.5. implementar \\
una estrategia de \\
bonos de descuentos \\
en mercancía \\
por servicios \\
$\begin{array}{l}\text { de reparación y } \\
\text { mantenimiento }\end{array}$
\end{tabular}

\section{Elaboración propia}

Una vez realizado el análisis DOFA, se identificó la existencia de deficiencias operativas del producto por una mala gestión de inventarios, que incluye la no existencia de una política de inventarios, no control de almacenes, ni del 
De acuerdo con el análisis FODA se tienen los siguientes resultados:

- Estrategias (FO): Relacionadas a una redistribución del área de almacenamiento, aumentar Nivel de servicio y gestión de inventarios.

- Estrategias (DO): Enfocadas al diseño de un modelo de inventarios que establezca una política clara en cuanto al layout de almacenes; plan de entrenamiento que garantice personal idóneo y conocimiento técnico del proceso y servicio.

- Estrategias (FA): Considerar acciones encaminadas a diseñar y fortalecer el servicio, de lo contrario los clientes pueden migrar a la competencia; así como buscar nuevos proveedores que permitan bajar costos de compras y renegociar los mismos.

- Estrategias (DA): Los agotados representan una gran amenaza no solo para el servicio sino para continuidad del negocio, de aquí la importancia del modelo a seguir en la gestión de inventarios.

\section{Diagnostico VESTER}

"Es una herramienta de análisis para establecer causas y consecuencias de un problema; fue desarrollada por el alemán Frederic Vester y aplicada con éxito en diversos campos; su método de análisis permite identificar un problema en doble vía, es decir su incidencia de este sobre problemas y la incidencia que otros problemas tienen sobre él”. (Chaparro A, 2000).

Para desarrollar este método, se partió de la confrontación de problemas causados por la mala gestión de inventarios de la empresa; de acuerdo con lo anterior se tiene la siguiente secuencia para establecer la valoración de acuerdo con los parámetros establecidos por el método como se muestra en la tabla 5 y 6.
Tabla 5. Escala de valoración

\begin{tabular}{lc}
\hline \multicolumn{1}{c}{ Relación de causalidad } & Valor \\
\hline Es causa MUY DIRECTA & 3 \\
Es causa MEDIANAMENTE & 2 \\
DIRECTA 2 & 1 \\
Es causa INDIRECTA 1 & 0 \\
NO es causa & Elaboración propia
\end{tabular}

La tabla 5 describe la escala de la valoración empleada para la causa de los problemas.

Tabla 6. Pasivos y activos VESTER

\begin{tabular}{lcc}
\hline & Pasivos (Y) & Activos (X) \\
\hline Almacén & 14 & 15 \\
Compras & 12 & 13 \\
Despacho & 11 & 13 \\
Servicio & 12 & 13 \\
Recurso humano & 9 & 7 \\
$\begin{array}{l}\text { Sistematización } \\
\text { información }\end{array}$ & 12 & 9 \\
\hline \multicolumn{3}{c}{ Elaboración propia }
\end{tabular}

La tabla 6 es el resultado de la ponderación de pasivos y activos, con los cuales se graficaron para determinar en qué parte del cuadrante se encuentra ubicado el problema como se muestra en la figura 1.

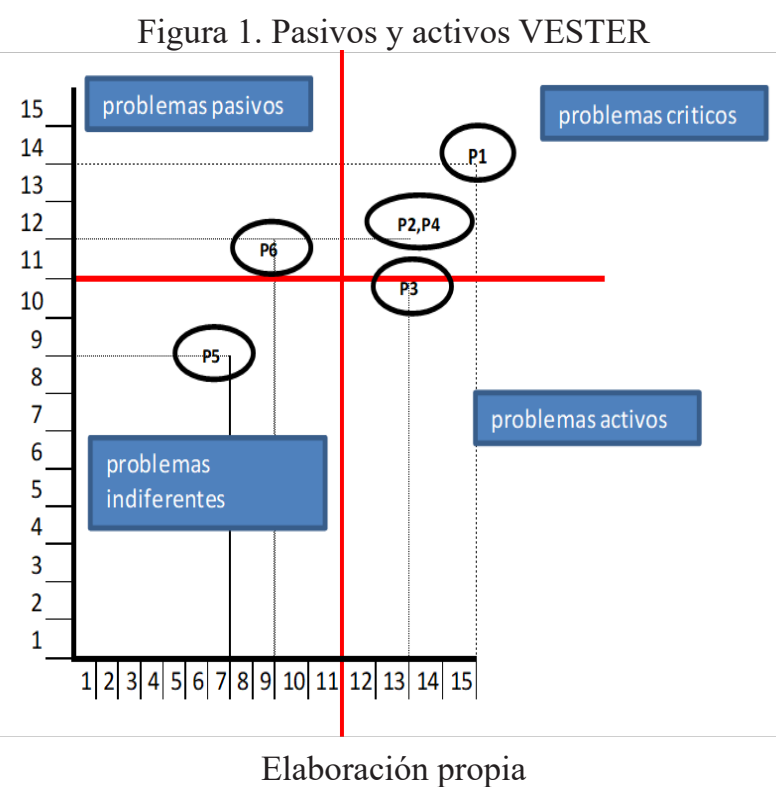


Con respecto al cuadrante de problemas críticos, se identifican los códigos P1, P2, P3 Y P4 los cuales corresponden a almacén, compras, despacho y servicio. En cuento al cuadrante de problemas pasivos se identifica sistematización de la información, lo cual indica poca inferencia causal, sin embargo, al intervenir los críticos deberían mermar los pasivos. Es decir, "se fundamenta en la comprensión e interpretación teórica de la investigación a partir de la formulación de una hipótesis de trabajo; busca identificar la asociación entre los temas a estudiar y la evidencia científica existente". (Anckerman \& Cheesman, 2010).

De acuerdo con el análisis el método para llevar a cabo el inventario incluye:

a. Inventario físico de referencias.

b. Registro en plantilla en Excel (Ver figura 2)

Figura 2. Registro en Excel

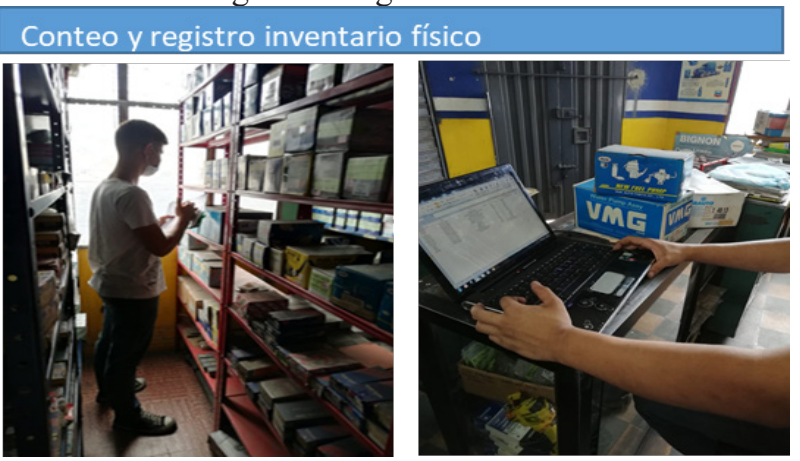

Elaboración propia

c. Implementación método $\mathrm{ABC}$ para clasificación de inventarios y redistribución en áreas de almacenamiento (ver figura 3)

Figura 3. Clasificación ABC
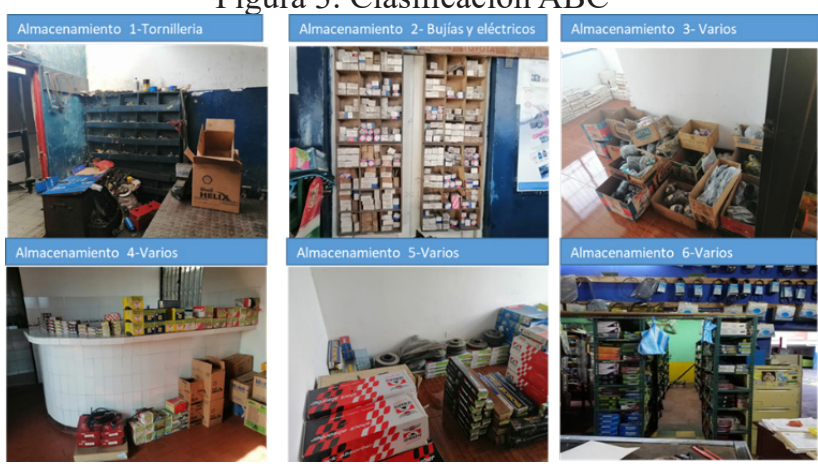

Elaboración propia d. Marcación y rotulación de producto (Ver figura 4)

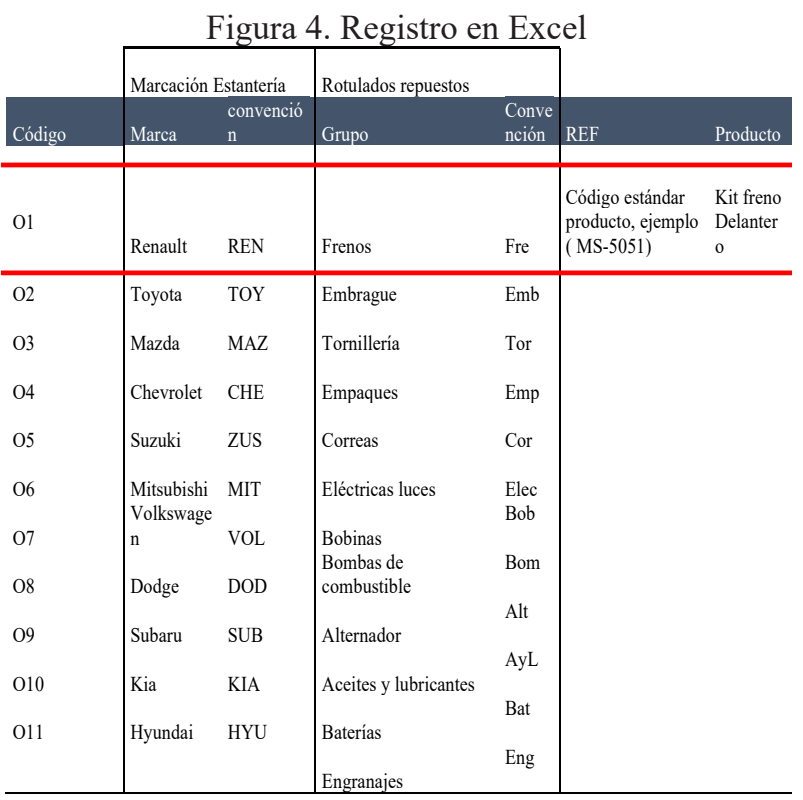

Elaboración propia

El registro de la matriz como se muestra en la figura 4 contiene código, marca, grupo para lograr un adecuado almacenamiento en las estanterías; esta rotulación implica una distribución de elementos los cuales se indican en la figura 5 .

Figura 5. Estructura de la rotulación

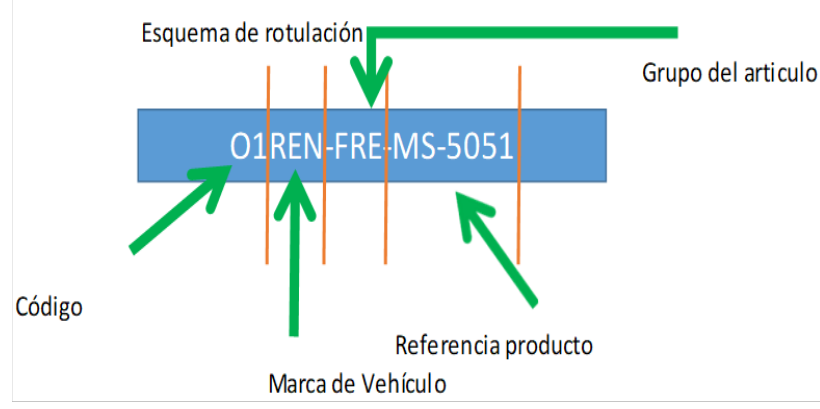

Elaboración propia

\section{Propuesta del sistema de gestión de inventarios para la empresa TÉCNITALLER S.A.S.}

De acuerdo con el diagnóstico que se le realizó a la empresa Tecnitaller S.A.S al sistema de gestión actual de inventarios, es necesario dinamizar la gestión de inventarios a través de la adquisición de un software que condense y gestione el manejo 
de entradas, el almacenamiento y salidas de los repuestos en la empresa como la facturación; siguiendo la estructura del sistema como se indica en la figura 6.

Figura 6. Estructura modular se software de gestión de inventarios

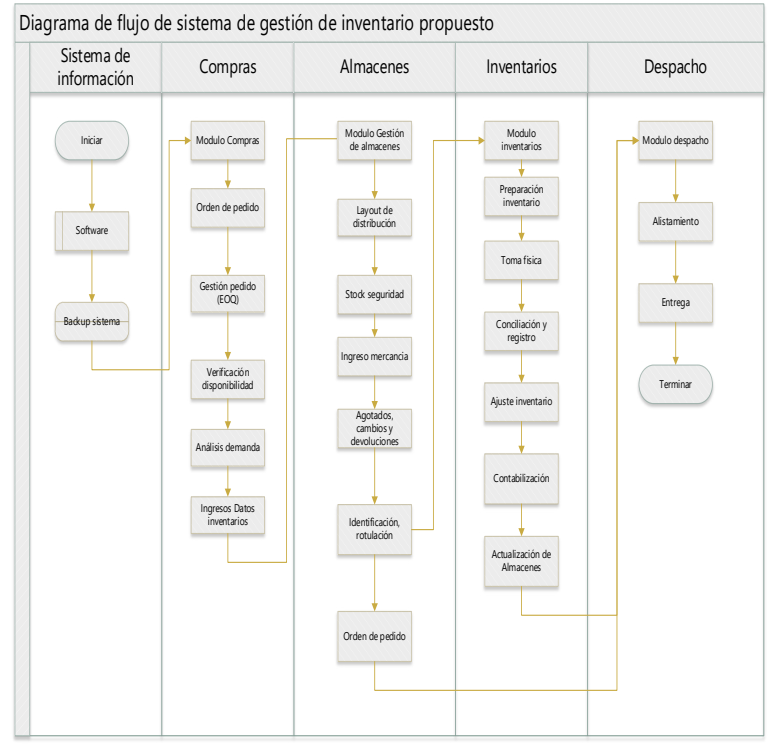

Elaboración propia

De acuerdo con la figura 6 plantea para la gestión de inventarios:

- Módulo de compras: Compuesto de los procesos de Orden y gestión del pedido, verificación del pedido, análisis de demanda,

- Módulo de Almacenes: Se compone de los procesos de layout, stock de seguridad, ingreso mercancía, agotados, cambios y devoluciones, cambios y devoluciones. Módulo de Inventario: Incluye, preparación de inventarios, tomas físicas, conciliación y registro, ajuste y contabilización.

- Módulo de Despacho: incluye alistamiento y entrega.

\section{Análisis económico del sistema de gestión de inventarios aplicar Técnitaller S.A.S}

El análisis económico que se planteó para el proyecto en la implementación del sistema de gestión de inventarios para la empresa Tecnitaller S.A.S, contiene la evaluación de costos actuales del proceso como se muestra en la tabla 7 .
Tabla 7. Costos actuales del proceso

\begin{tabular}{ll}
\hline \multicolumn{1}{c}{ Concepto } & \multicolumn{1}{c}{$\$$} \\
\hline Costo de pedir & $\$ 10.187 .373$ \\
costo de mantener & $\$ 5.201 .158$ \\
Costo stock seguridad & $\$ 1.209 .915$ \\
Costo obsolescencia & $\$ 345.000$ \\
Costos faltantes & $\$ 934.000$ \\
\hline Total, Costo & $\$ 17.877 .445$ \\
\hline \multicolumn{2}{c}{ Elaboración propia }
\end{tabular}

Los costos actuales del proceso en función de la demanda de repuestos por categorías, son de \$17.877.445 / mes; sin embargo, el costo de implementar a partir de un software es de $\$ 21.595 .150$, lo cual es altamente beneficioso si se parte del hecho de que al proyectar los costos actuales al año se tendría un incremento alto con relación a las ventajas que otorga un proceso a tres meses con la ventaja de sistematizar los procesos, aspectos legales y financieros para el negocio; de acuerdo con lo anterior en la tabla 8 se muestran los costos de la implementación del sistema de gestión de inventarios.

Tabla 8. Costos de implementación propuesta

\begin{tabular}{lcr}
\hline \multicolumn{1}{c}{ Concepto } & $\$$ & \\
\hline Paquete Software & $\$$ & 4.885 .000 \\
Tecnologías y equipos & $\$$ & 9.898 .700 \\
Recurso humano instalación y parametrización software & $\$$ & 3.800 .000 \\
Papelería y útiles oficina & $\$$ & 134.460 \\
Recursos humanos para digitación & $\$$ & 1.514 .210 \\
recurso humano entrenamiento y capacitación & $\$$ & 1.362 .780 \\
\hline \multicolumn{1}{c}{ Total, costo proyecto } & $\$$ & 21.595 .150 \\
\hline
\end{tabular}

Elaboración propia

\section{CONCLUSIONES}

De acuerdo con el objetivo específico de realizar un diagnóstico de situación actual de la empresa; para su realización se emplearon herramientas de análisis estratégico como la de DOFA Y VESTER, las cuales junto con la lista de chequeo permitieron identificar las problemáticas asociadas a la gestión actual de la empresa Técnitaller S.A.S, dentro de las que se destacan, la falta de control y monitoreo de los inventarios, mala rotación de la mercancía, 
bajo nivel de servicio, no manejo de stock de reposición, almacenamiento no controlado y descentralizado entre otros factores; estos hallazgos permitieron elaborar una propuesta de mejora que incluyo la cadena logística completa con miras a tener un control proceso que mejorara la gestión interna en la empresa; por lo tanto, dicho objetivo específico propuesto se cumplió.

En lo que respecta al objetivo de elaborar una propuesta de gestión inventarios, una vez realizado y analizado el diagnostico, se definió un modelo el cual parte de una clasificación $\mathrm{ABC}$ de referencia, así como la estimación de stock de seguridad, centralización y optimización de layout de almacenamiento; dicha propuesta se condensa en el diseño de los módulos necesarios y ajustados a la misión de la empresa, lo cuales comprenden la gestión de compras, almacén, inventarios y despachos, los cuales constituyen la actividad principal y diaria misional de la empresa; por lo tanto a partir de dicha propuesta se dio cumplimiento con el objetivo propuesto.

De acuerdo con el estudio económico de la propuesta esta tiene un valor total para la empresa (incluyendo compra de software, costos de implementación) de \$21.595.150; este valor incluye el valor del paquete y los costos de implementación; desde el punto de vista de la viabilidad en su implementación, se puede concluir que sus beneficios operativos y económicos de sus implementación hacen que sea viable la propuesta frente a los actuales costos que demanda la gestión actual de inventarios en la empresa Técnitaller S.A.S

\section{REFERENCIAS}

1. Anckerman , S., \& Cheesman , S. (2010). Marco Teorico - Elaborado con fines docentes. Guatemala: Repositorios academicos- Universidad de San carlos de guatemala. Obtenido de https://investigar1.files.wordpress.com/2010/05/marco-e-hipotesis-investigacion.pdf

2. Anónimo. (2012). Rotacion de inventarios. Obtenido de https://repository.unilibre.edu.co/ bitstream/handle/10901/9385/Proyecto\%20de\%20grado\%20(2).pdf?sequence=1\&isAllowed=y

3. Arguello Lopez, G. M., \& Fragoso Serrano, E. F. (Noviembre de 2015). ResearchGate. Obtenido de Diagnóstico de la gestión de inventarios de las pequeñas y medianas empresas del sector industrial del área metropolitana de Bucaramanga: https://www.researchgate.net/publication/284732574 Diagnostico_de_la_gestion_de_inventarios_de_las_pequenas_y_medianas_empresas_del_ sector_industrial_del_area_metropolitana_de_Bucaramanga

4. Bravo, O. (1981). Contabilidad de costos. Academia.edu. Obtenido de https://www.academia. edu/25271370/Contabilidad_de_costos_oscar_gomez_bravo

5. Buenaño del pezo , w. G., \& Jimenez Merino, D. H. (2016). Diseño de modelo de control de inventarios para la empresa Produbal S.A. Guayaquil: Repositorios academicos de la universidad de guayaquil. Obtenido de http://repositorio.ug.edu.ec/bitstream/redug/14620/1/TESIS\%20Cpa\%20 147\%20-\%20Dise $\%$ C3\%B10\%20de\%20modelo $\% 20 \mathrm{de} \% 20$ control $\% 20 \mathrm{de} \% 20$ inventarios $\% 20$ para\%201a\%20Empresa\%20Produbal.pdf

6. Chaparro A, O. (2000). Analisis y priorización de problemas. Bogotá: Corpoica.

7. Contreras, M. (2011). Educapuntes. Obtenido de Antecedentes de la investigación : http:// educapuntes.blogspot.com/2011/04/antecedentes-de-la-investigacion.html

8. Elwood, B. (2000). Sistema de produccion e inventario . San Nicolas de los garza: Repositorios academicos Universidad autonomo de nuevo leon. Obtenido de http://cdigital.dgb.uanl.mx/ te/1020130058.pdf

9. Gestión de operaciones. (2021). Gestión de operaciones.net. Obtenido de https://www. gestiondeoperaciones.net/gestion-de-calidad/que-es-el-diagrama-de-ishikawa-o-diagrama-decausa-efecto/

10. Gutiérrez, V., \& Vidal, C. J. (2007). Modelos de Gestión de Inventarios en Cadenas. Medellin (Colombia) - Cali (Colombia): Universidad de Antioquia y Universidad del valle. Obtenido de http://www.scielo.org.co/pdf/rfiua/n43/n43a12.pdf

11. Narasemban, S. (1996). Planeación de la produccion y control. Repositorios academicos. Obtenido 
de http://cdigital.dgb.uanl.mx/te/1020130058.pdf

12. Naula Valla, S. M. (2018). Control de inventarios y su incidenica en los estados financieros. Guayaquil Ecuador: Eumed.net. Obtenido de https://www.eumed.net/rev/oel/2018/11/inventariosestados-financieros.html

13. Orosco Fábian, J. R. (2009). Antecedentes de la investigación. Riofa Ponencias PDF. Obtenido de Antecedentes de la investigación: https://riofaponencias.files.wordpress.com/2012/04/ antecedentes.pdf

14. Remache Coraizaca, M. F. (2017). Diseño de modelo de gestión de inventarios basado en el metodo $A B C$ en la ferreteria Ferrimag del canton la troncal. Cantón la Troncal: Repositorios academicos de la universidad Catolica de cuenca. Obtenido de http://dspace.ucacue.edu.ec/bitstream/ reducacue $/ 7636 / 1 /$ estudio $\% 20$ de $\% 20$ caso $\% 20 \_\% 20$ dise $\%$ c3\%91o\%20de $\% 20$ modelo $\% 20 \mathrm{de} \% 20$ gestion $\% 20 \mathrm{de} \% 20$ inventarios $\% 20$ basado $\% 20$ en $\% 20 \mathrm{el} \% 20 \mathrm{~m} \% \mathrm{c} 3 \% 89$ todo $\% 20 \mathrm{abc} \% 20 \_\% 20$ maria\%20remache.pdf

15. Rincón M, L. N. (2016). Sistema de control interno para el área contable y el area financiera de la empresa productora avicola. Bogotá: UlaSalle.

16. Robles, A. Y. (2008). Control de inventarios con analisis de demanda. Lima: Repositorios academicos. Obtenido de https://repository.unilibre.edu.co/bitstream/handle/10901/9385/ Proyecto\%20de \%20grado\%20(2).pdf?sequence=1\&isAllowed=y

17. Sistema de produccion de inventarios. (2010). Control de inventarios. Facultad de estudio a distancia - Universidad. Obtenido de http://virtual.umng.edu.co/distancia/ecosistema/ovas/ administracion_empresas/logistica/unidad_3/DM.pdf

18. SPCgroup. (2021). SPCgroup. Obtenido de SPCgroup: https://spcgroup.com.mx/diagrama-deishikawa/

19. Torres, M. M. (2008). Gestion de stock. España: Repositorios academicos. Obtenido de https:// repository.unilibre.edu.co/bitstream/handle/10901/9385/Proyecto\%20de\%20grado\%20(2). pdf? sequence $=1$ \&isAllowed $=\mathrm{y}$

20. Zapata Cortes, J. A. (2014). Fundamentos de la gestion de inventarios. Medellin: Esumer Institución universitaria. Obtenido de https://www.esumer.edu.co/images/centroeditorial/Libros/ fei/libros/Fundamentosdelagestiondeinventarios.pdf 